\title{
Efficacy and Outcomes of Lianhuaqingwen Capsule for Coronavirus Disease 2019: A Retrospective Propensity Score Matching Analysis
}

zhiyong liu ( $\sim$ liuzy271@163.com )

Department of Gastroenterology, 920th Hospital of the PLA Joint Logistics Support Force, Kunming,Yunnan650032, China. https://orcid.org/0000-0002-7135-2746

\section{Yongsheng Du}

Department of Gastroenterology, 920th Hospital of the PLA Joint Logistics Support Force, Kunming,Yunnan650032, China.

Jiankun Liu

Department of Gastroenterology, 920th Hospital of the PLA Joint Logistics Support Force, Kunming,Yunnan650032, China.

\section{Xiaolong Wei}

Department of Gastroenterology, 920th Hospital of the PLA Joint Logistics Support Force, Kunming,Yunnan650032, China.

\section{Feiyang Zheng}

Department of Gastroenterology, 920th Hospital of the PLA Joint Logistics Support Force, Kunming,Yunnan650032, China.

\section{Raomei LI}

Department of Gastroenterology, 920th Hospital of the PLA Joint Logistics Support Force, Kunming,Yunnan650032, China.

\section{Shurong Zhang}

Department of Gastroenterology, 920th Hospital of the PLA Joint Logistics Support Force, Kunming,Yunnan650032, China.

\section{Research}

Keywords: Coronavirus disease 2019,Lianhuaqingwen Capsule, Clinical efficacy, Outcomes

Posted Date: August 11th, 2020

DOI: https://doi.org/10.21203/rs.3.rs-55928/v1

License: (c) (i) This work is licensed under a Creative Commons Attribution 4.0 International License. Read Full License 


\section{Abstract}

Background: Coronavirus disease 2019 (COVID-19) has outbroken in Wuhan, China, in December, 2019, and became a global pandemic.No effective antiviral agents were approved for COVID-19. In this study, we aim to evaluate the efficacy and outcomes of Lianhuaqingwen (LH) capsulein patients with COVID-19.

Methods:In this retrospective cohort study, we included 147 hospitalized patients with laboratoryconfirmed severe acute respiratory syndrome coronavirus 2 (SARS-CoV-2)infection between February $4^{\text {th }}$ and April $15^{\text {th }}, 2020$, in Wuhan Huoshen shan hospital. Patients were grouped as usual treatment alone or combination with LH capsules. Propensity score matching analysis were performed between two groups. Primary outcomes were mortality and duration of hospitalization time. Secondary outcomes included the recovery rate of chest radiological manifestations and abnormal laboratory examinations.

Results: In two groups, 40 pairs patients were matched for analysis. The baseline information and clinical characteristics were comparable. 73 (91.25\%) of all 80 patients were older than 50 years old. All patients in LH treatment group and $38(95 \%)$ in usual treatment group were surviving respectively $(P=0.494)$. The hospitalization time of patients in LH treatment group was significantly shorter than usual treatment group (17.85 \pm 6.612 vs. $20.72 \pm 5.01$ days, $P=0.032$ ). After treatment, the rate of lung lesions detected by chest computerized tomography (CT) was lower in LH treatment group ( $30 \%$ vs. $55.0 \%, \mathrm{P}=0.024)$.

Conclusion: LH capsules were associated with the improved clinical effects, and might be recommended in the treatment practices of COVID-19 patients. Moreover, further evaluation of LH capsules in large population randomized controlled trial is needed.

\section{Background}

Since coronavirus disease 2019 (COVID-2019) first outbroke in December 2019 in Wuhan, China, this epidemic has rapidly spread globally[1]. As of 15 July 2020, according to World Health Organization (WHO) report, 13,150,645 patients of COVID-19 were laboratory-confirmed, and 574,464 deaths globally (https://www.who.int). The rapid outbreak worldwide might reflect population are generally susceptible to the pathogen of COVID-19, severe acute respiratory syndrome coronavirus 2 (SARS-CoV-2)[2, 3]. The origin of the SARS-CoV-2 is still unknown. Sequencing analysis of SARS-CoV-2 showed its genome sequence is highly related with bat-derived SARS-like coronavirus, and has $88 \%$ nucleotide sequence homology, the receptor-binding domain structure of SARS-CoV-2 is similar to that of SARS-CoV[4]. The epidemiological and clinical characteristics of patients with COVID-19 had been described previously, such as familial clustering outbreak, fever, dry cough, and lymphopenia[3, 5]. In China, mortality of COVID2019 was reported to be nearly $4 \%-7.5 \%[3,6]$, and older patients with comorbidities were more likely to be critical ill and admitted to the intensive care unit (ICU), whose mortality increased to $51.7 \%-61.5 \%[6,7]$. Thus, management of patients is cardinal to control COVID-19 adverse impacts, especially for those severe patients. 
Several therapeutic interventions and medications for COVID-19 patients have been adopted during the outbreak period of this epidemic[8]. Remdesivir is a broad-spectrum antiviral agent for several RNA viruses[9], and developed for Ebola virus infection[10]. Remdesivir was applied to the first confirmed patient of COVID-2019 in the United States and showed improved clinical conditions by alleviating lung viral loads and improving lung function[11]. Chloroquine was a widely used antimalarial drug and exerted strong antiviral effects by increasing endosomal $\mathrm{pH}$ required for virus/cell fusion and interfering with the glycosylation of cellular receptors of SARS-CoV[12]. Several multicenter clinical trials conducted in China showed that chloroquine have apparent efficacy to decrease the exacerbation of COVID-19 pneumonia without severe side effects[13]. Chloroquine is also recommended to be included in the Diagnosis and Treatment Protocol for Coronavirus Pneumonia (Trial version 7) issued by the National Health Commission of China. Other recommended therapy methods in this protocol include interferon, corticosteroids, immunotherapy and traditional Chinese medications[8, 14]. But no effective antiviral drugs were approved and the potential vaccines for COVID-19 are still in clinical trials $[15,16]$.

Lianhuaqingwen (LH) capsule (Yiling Pharmaceutical Corp, Shijiazhuang, China) is a traditional Chinese medicine formula, and consists of 13 natural herbs. LH capsule has been widely used in influenza viruses and showed broad-spectrum antiviral effects by ameliorating cardinal symptoms and reducing the virus infection course[17, 18]. In vitro study, li et al. found LH capsule significantly inhibited SARS-CoV-2 replication and exerted anti-inflammatory functions in Vero E6 cells[19]. Moreover, a multicenter randomized controlled trial indicated LH capsule could promote symptom recovery of COVID-19 patients and reduce the duration of viral shedding[20]. In the present study, we conducted a retrospective study to evaluate the efficacy and outcomes of LH capsule for patients with COVID-19 in Wuhan Huoshenshan hospital by propensity score matching (PSM) analysis.

\section{Methods}

\section{Study design and patient selection}

This retrospective cohort study includes patients who were diagnosed with COVID-19 infection between February 4th and April 15th, 2020, in Wuhan Huoshenshan Hospital. The study has been approved by the ethic committee of Wuhan Huoshenshan Hospital and 920th Hospital of the PLA Joint Logistics Support Force, and registered with Chinese Clinical Trial Registry website (www.chictr.org/cn/, No:

ChiCTR2000035046).

In this study, we recruited 147 patients aged 18 years or older and laboratory-confirmed with COVID-19 by nucleic acid assay[14]. Exclusion criteria included: 1) patients aged $₫ 18$ years old; 2 ) missing pathogen or chest computerized tomography (CT) detection; 3 ) severe comorbidities, such as advanced malignancy, congenital heart disease or autoimmune diseases. We recorded demographic information, clinical characteristics, medical histories, laboratory data, outcomes and mortality of the selected patients. 


\section{Procedures}

All the eligible patients received appropriate usual treatment according to The Protocol for Diagnosis and Treatment of Novel Coronarvirus Pneumonia (version 5) (National Health Commission of China). Usual treatment methods generally included supportive treatment such as oxygen inhalation, antiviral therapy, antibiotic therapy and traditional Chinese medications. Lianhuaqingwen (LH) capsules were supplied by manufacture. In LH treatment group, patients with COVID-19 received usual treatment and LH capsules at a dose of $1.4 \mathrm{~g}$ thrice daily. Patients received routine treatment without LH capsules were classified as usual treatment group. Meanwhile, the vital clinical signs, blood testing, chest CT and adverse events were monitored.

\section{Propensity Score Matching}

Propensity score matching (PSM) is an effective statistical tool for reducing selection biases[21]. In this study, we performed PSM analysis to balance the baseline covariates between two treatment groups using the nearest neighbor method with a 1:1 matching ratio. The matched variables of the patients in two groups included age, gender (male, female), duration time from symptom onset to hospitalization, comorbidities (hypertension, diabetes, coronary heart disease, pulmonary disease and other chronic diseases), clinical symptoms (fever, highest temperature, cough, expectoration, dyspnea, chest distress, myalgia, headache, palpitation, diarrhea, nausea, SpO2), lung lesions detected by chest CT and treatment methods. Balance between two groups was defined as a $\mathrm{P}$ value $>0.05$.

\section{Outcomes}

The primary outcomes for this study were mortality and hospitalization time. Hospital discharge criteria of COVID-19 patients complied with The Protocol for Diagnosis and Treatment of Novel Coronarvirus Pneumonia (version 5) (National Health Commission of China). Hospital discharge criteria were as following: 1) temperature returns to normal more than 3 days; 2 ) respiratory symptoms recovered significantly; 3) two consecutive nucleic acid assay detections for respiratory tract specimens were negative.

Secondary outcomes were the recovery rate of radiological and laboratory examinations which included the proportion of lung lesions on chest $\mathrm{CT}$, blood routine examination, incidence of abnormal coagulation function, blood biochemistry parameters and C-reactive protein level.

\section{Statistical analysis}

Continuous variables were presented as mean \pm standard deviation (sd), and compared with independent $t$ test. Categorical variables were described as $n(\%)$ and compared by $\chi^{2}$ or Fisher's exact test. Statistical analyses were performed with SPSS (version 22.0) (SPSS Inc, Chicago, USA). Separately, we conducted 
Gehan-Breslow-Wilcoxon test to compare the discharge rate between two groups, and drew the hospital discharge plot by GraphPad Prism version (GraphPad Corp, California, USA). All statistical analyses were 2-tailed, and $P$ value $<0.05$ was considered to be significant.

\section{Results}

\section{Clinical characteristics and treatment methods}

151 patients with COVID-19 at Huoshenshan hospital included in our study, 4 patients were excluded due to age $<18$ years, the lack of SARS-CoV-2 nucleic acid assay and malignancy. Therefore, a total of 147 patients met the eligibility criteria, including 79 in LH treatment group (53.7\%) and 68 in usual treatment group (46.3\%). After PSM analysis, 40 pairs patients were matched between two groups. Flow chart of this study is presented in Fig. 1. 
Table 1

The baseline characteristics and clinical symptoms of patients before and after PSM.

\begin{tabular}{|c|c|c|c|c|c|c|}
\hline \multirow{3}{*}{ Basic information } & \multicolumn{3}{|c|}{ Before PSM } & \multicolumn{3}{|l|}{ After PSM } \\
\hline & $\begin{array}{l}\mathrm{LH} \\
\text { treatment } \\
\text { group }\end{array}$ & $\begin{array}{l}\text { Usual } \\
\text { treatment } \\
\text { group }\end{array}$ & $\begin{array}{l}P \\
\text { value }\end{array}$ & $\begin{array}{l}\text { LH } \\
\text { treatment } \\
\text { group }\end{array}$ & $\begin{array}{l}\text { Usual } \\
\text { treatment } \\
\text { group }\end{array}$ & $\begin{array}{l}\mathrm{P} \\
\text { value }\end{array}$ \\
\hline & $\begin{array}{l}(n= \\
79,53.7 \%)\end{array}$ & $\begin{array}{l}(n= \\
68,46.3 \%)\end{array}$ & & $\begin{array}{l}(n= \\
40,50 \%)\end{array}$ & $\begin{array}{l}(n= \\
40,50 \%)\end{array}$ & \\
\hline Age (years, $x \pm s d)$ & $\begin{array}{l}64.81 \pm \\
10.66\end{array}$ & $\begin{array}{l}59.79 \pm \\
12.805\end{array}$ & 0.012 & $\begin{array}{l}63.83 \pm \\
11.325\end{array}$ & $\begin{array}{l}62.75 \pm \\
12.066\end{array}$ & 0.682 \\
\hline Age $\geq 50$ years, $n(\%)$ & $\begin{array}{l}71 \\
(89.9 \%)\end{array}$ & $\begin{array}{l}56 \\
(82.4 \%)\end{array}$ & 0.185 & $\begin{array}{l}36 \\
(90.0 \%)\end{array}$ & $\begin{array}{l}37 \\
(92.5 \%)\end{array}$ & 1.000 \\
\hline Males, n (\%) & $\begin{array}{l}43 \\
(54.4 \%)\end{array}$ & $\begin{array}{l}28 \\
(41.2 \%)\end{array}$ & 0.109 & $\begin{array}{l}16 \\
(54.4 \%)\end{array}$ & $\begin{array}{l}18 \\
(41.2 \%)\end{array}$ & 0.651 \\
\hline Females, n (\%) & $\begin{array}{l}36 \\
(45.6 \%)\end{array}$ & $\begin{array}{l}40 \\
(58.8 \%)\end{array}$ & & $\begin{array}{l}24 \\
(60.0 \%)\end{array}$ & $\begin{array}{l}22 \\
(55.0 \%)\end{array}$ & \\
\hline $\begin{array}{l}\text { Duration time from symptom } \\
\text { onset to hospitalization } \\
\text { (days, } x \pm \text { sd) }\end{array}$ & $\begin{array}{l}16.46 \pm \\
9.099\end{array}$ & $\begin{array}{l}16.35 \pm \\
8.753\end{array}$ & 0.945 & $\begin{array}{l}18.10 \pm \\
8.825\end{array}$ & $\begin{array}{l}16.78 \pm \\
10.078\end{array}$ & 0.533 \\
\hline \multicolumn{7}{|l|}{ Comorbidities } \\
\hline Hypertension, n (\%) & $\begin{array}{l}35 \\
(44.3 \%)\end{array}$ & $\begin{array}{l}19 \\
(27.9 \%)\end{array}$ & 0.04 & $\begin{array}{l}12 \\
(30.0 \%)\end{array}$ & $\begin{array}{l}12 \\
(30.0 \%)\end{array}$ & 1.000 \\
\hline Diabetes, n (\%) & $\begin{array}{l}17 \\
(21.5 \%)\end{array}$ & $8(11.8 \%)$ & 0.117 & $9(22.5 \%)$ & $8(20.0 \%)$ & 0.785 \\
\hline CHD, n (\%) & $4(5.1 \%)$ & $4(5.9 \%)$ & 0.827 & $3(7.5 \%)$ & $2(5.0 \%)$ & 1.000 \\
\hline Pulmonary disease, n (\%) & $3(3.8 \%)$ & $1(1.5 \%)$ & 0.387 & $0(0.0 \%)$ & $1(2.5 \%)$ & 1.000 \\
\hline Other chronic diseases, $\mathrm{n}(\%)$ & $\begin{array}{l}28 \\
(35.4 \%)\end{array}$ & $\begin{array}{l}15 \\
(22.4 \%)\end{array}$ & 0.085 & $\begin{array}{l}13 \\
(32.5 \%)\end{array}$ & $\begin{array}{l}13 \\
(32.5 \%)\end{array}$ & 1.000 \\
\hline \multicolumn{7}{|l|}{ Clinical symptoms } \\
\hline Fever, n (\%) & $\begin{array}{l}74 \\
(93.7 \%)\end{array}$ & $\begin{array}{l}61 \\
(89.7 \%)\end{array}$ & 0.381 & $\begin{array}{l}33 \\
(82.5 \%)\end{array}$ & $\begin{array}{l}27 \\
(67.5 \%)\end{array}$ & 0.121 \\
\hline $\begin{array}{l}\text { Highest temperature }\left({ }^{\circ} \mathrm{C},{ }^{\prime} \mathrm{x} \pm\right. \\
\text { sd) }\end{array}$ & $\begin{array}{l}38.14 \pm \\
0.981\end{array}$ & $\begin{array}{l}37.92 \pm \\
1.050\end{array}$ & 0.189 & $\begin{array}{l}38.153 \pm \\
0.946\end{array}$ & $\begin{array}{l}37.937 \pm \\
1.637\end{array}$ & 0.367 \\
\hline Cough, n (\%) & $\begin{array}{l}54 \\
(68.4 \%)\end{array}$ & $\begin{array}{l}49 \\
(72.1 \%)\end{array}$ & 0.625 & $\begin{array}{l}32 \\
(80.0 \%)\end{array}$ & $\begin{array}{l}31 \\
(77.5 \%)\end{array}$ & 0.785 \\
\hline Expectoration, n (\%) & $7(8.9 \%)$ & $6(8.8 \%)$ & 0.994 & $5(12.5 \%)$ & $4(7.5 \%)$ & 0.712 \\
\hline
\end{tabular}

PSM, propensity score matching; LH, lianhuaqingwen capsule; CHD, coronary heart disease 


\begin{tabular}{|c|c|c|c|c|c|c|}
\hline \multirow[b]{2}{*}{ Dyspnea, n (\%) } & \multicolumn{3}{|c|}{ Before PSM } & \multicolumn{3}{|l|}{ After PSM } \\
\hline & $\begin{array}{l}27 \\
(34.2 \%)\end{array}$ & $\begin{array}{l}38 \\
(55.9 \%)\end{array}$ & 0.008 & $\begin{array}{l}17 \\
(42.5 \%)\end{array}$ & $\begin{array}{l}19 \\
(47.5 \%)\end{array}$ & 0.653 \\
\hline Chest distress, n (\%) & $3(3.8 \%)$ & $7(10.3 \%)$ & 0.119 & $2(5.0 \%)$ & $4(10.0 \%)$ & 0.657 \\
\hline Myalgia, n (\%) & $\begin{array}{l}31 \\
(39.2 \%)\end{array}$ & $\begin{array}{l}32 \\
(47.1 \%)\end{array}$ & 0.340 & $\begin{array}{l}18 \\
(45.0 \%)\end{array}$ & $\begin{array}{l}19 \\
(47.5 \%)\end{array}$ & 0.823 \\
\hline Headache, n (\%) & $8(10.1 \%)$ & $0(0.0 \%)$ & 0.007 & $1(2.5 \%)$ & $0(0.0 \%)$ & 1.000 \\
\hline Palpitation, n (\%) & $2(2.5 \%)$ & $3(4.4 \%)$ & 0.531 & $1(2.5 \%)$ & $2(5.0 \%)$ & 1.000 \\
\hline Diarrhea, n (\%) & $3(3.8 \%)$ & $9(13.2 \%)$ & 0.037 & $3(7.5 \%)$ & $2(5.0 \%)$ & 1.000 \\
\hline Nausea, n (\%) & $\begin{array}{l}12 \\
(15.2 \%)\end{array}$ & $\begin{array}{l}10 \\
(14.7 \%)\end{array}$ & 0.935 & $7(17.5 \%)$ & $3(7.5 \%)$ & 0.311 \\
\hline SpO2 (\%,'x \pm sd) & $\begin{array}{l}96.22 \pm \\
3.225\end{array}$ & $\begin{array}{l}96.82 \pm \\
1.727\end{array}$ & 0.149 & $\begin{array}{l}96.25 \pm \\
2.519\end{array}$ & $\begin{array}{l}95.73 \pm \\
3.776\end{array}$ & 0.467 \\
\hline Lung lesions, n (\%) & $\begin{array}{l}78 \\
(98.7 \%)\end{array}$ & $\begin{array}{l}66 \\
(97.1 \%)\end{array}$ & 0.596 & $\begin{array}{l}38 \\
(95.0 \%)\end{array}$ & $\begin{array}{l}39 \\
(97.5 \%)\end{array}$ & 1.000 \\
\hline
\end{tabular}

Baseline characteristics of patients were list in Table 1. Before PSM, the age of patients in LH treatment group was higher than usual treatment group ( $64.81 \pm 10.66$ vs. $59.79 \pm 12.805$ years, $P=0.012)$. More patients had hypertension in LH treatment group (44.3\% vs. $27.9 \%, P=0.04)$. Smaller proportion of patients suffered from dyspnea $(34.2 \%$ vs. $55.9 \%, P=0.008)$ and diarrhea $(3.8 \%$ vs. $13.2 \%, P=0.037)$ in $\mathrm{LH}$ treatment group. 8 patients had headache in LH treatment group, while no patient in usual treatment group $(10.1 \%$ vs. $0.0 \%, P=0.007)$. There were no significant differences between two groups with regard to gender, duration time from symptom onset to hospitalization, diabetes, coronary heart disease, pulmonary disease and other chronic diseases, fever, highest temperature, cough, expectoration, chest distress, myalgia, palpitation, nausea, SpO2, and lung lesions. Common clinical symptom of COVID-19 patients was fever, 74 (93.7\%) in LH treatment group and 61 (89.7\%) in usual treatment group. The highest temperatures in two groups were $38.14 \pm 0.981^{\circ} \mathrm{C}$ and $37.92 \pm 1.050{ }^{\circ} \mathrm{C}$ respectively $(P=0.189)$. Chest CT indicated more than $95 \%$ had lung lesions both in LH group and usual treatment group. After PSM, two groups were comparable in terms of the demographic characteristics, comorbidities and clinical symptoms. More than $90 \%$ patients $(90.0 \%, 92.5 \%)$ in two matched groups were older than 50 years. 
Table 2

The therapeutic interventions of patients before and after PSM.

\begin{tabular}{|c|c|c|c|c|c|c|}
\hline \multirow{3}{*}{ Treatment methods } & \multicolumn{2}{|l|}{ Before PSM } & \multicolumn{4}{|c|}{ After PSM } \\
\hline & $\begin{array}{l}\text { LH } \\
\text { treatment } \\
\text { group }\end{array}$ & $\begin{array}{l}\text { Usual } \\
\text { treatment } \\
\text { group }\end{array}$ & $\begin{array}{l}P \\
\text { value }\end{array}$ & $\begin{array}{l}\text { LH } \\
\text { treatment } \\
\text { group }\end{array}$ & $\begin{array}{l}\text { Usual } \\
\text { treatment } \\
\text { group }\end{array}$ & $\begin{array}{l}\mathrm{P} \\
\text { value }\end{array}$ \\
\hline & $\begin{array}{l}(n= \\
79,53.7 \%)\end{array}$ & $\begin{array}{l}(n= \\
68,46.3 \%)\end{array}$ & & $\begin{array}{l}(n= \\
40,50 \%)\end{array}$ & $\begin{array}{l}(n= \\
40,50 \%)\end{array}$ & \\
\hline $\begin{array}{l}\text { Oxygen inhalation, } n \\
\text { (\%) }\end{array}$ & 78 (98.7\%) & $64(94.1 \%)$ & 0.124 & $\begin{array}{l}39 \\
(97.5 \%)\end{array}$ & $38(95.0 \%)$ & 1.000 \\
\hline $\begin{array}{l}\text { Mechanical } \\
\text { ventilation, } \mathrm{n}(\%)\end{array}$ & $1(1.3 \%)$ & $0(0.0 \%)$ & 0.352 & $0(0.0 \%)$ & $0(0.0 \%)$ & 1.000 \\
\hline $\begin{array}{l}\text { Antiviral therapy, } n \\
(\%)\end{array}$ & $56(70.9 \%)$ & 27 (39.7\%) & $\llbracket 0.001$ & $\begin{array}{l}24 \\
(60.0 \%)\end{array}$ & $23(57.5 \%)$ & 0.820 \\
\hline $\begin{array}{l}\text { Antibiotic therapy, } n \\
\text { (\%) }\end{array}$ & $41(51.9 \%)$ & $27(39.7 \%)$ & 0.139 & $\begin{array}{l}18 \\
(45.0 \%)\end{array}$ & $20(50.0 \%)$ & 0.654 \\
\hline $\begin{array}{l}\text { Antitussive therapy, } n \\
\text { (\%) }\end{array}$ & 47 (59.5\%) & 38 (55.9\%) & 0.658 & $\begin{array}{l}20 \\
(50.0 \%)\end{array}$ & $21(52.5 \%)$ & 0.823 \\
\hline $\begin{array}{l}\text { Traditional Chinese } \\
\text { medication, } \mathrm{n}(\%)\end{array}$ & 71 (89.9\%) & $59(86.8 \%)$ & 0.557 & $\begin{array}{l}39 \\
(97.5 \%)\end{array}$ & $38(95.0 \%)$ & 1.000 \\
\hline
\end{tabular}

The main treatment methods for COVID-19 patients were shown in Table 2. Here, before PSM, the proportion of patients received antiviral therapy in LH treatment group was significantly higher than that in usual treatment group $(70.9 \%$ vs. $39.7 \%, \mathrm{P}<0.001)$. 78 patients $(98.7 \%)$ in LH treatment group and 64 $(94.1 \%)$ in usual treatment group received oxygen inhalation. More than $85 \%$ patients in two groups were given traditional Chinese medications. Only 1 patient in LH treatment group needed mechanical ventilation. After PSM, 24 (60.0\%) patients in LH treatment group and $23(57.5 \%)$ in usual treatment group received antiviral therapy $(P=0.820)$. No patient received mechanical ventilation in two groups.

\section{Primary Outcomes}


Table 3

Differences of outcomes and lung lesions rate between two groups.

\begin{tabular}{|c|c|c|c|}
\hline & $\begin{array}{l}\text { LH treatment group }(n= \\
40,50 \%)\end{array}$ & $\begin{array}{l}\text { Usual treatment group }(n= \\
40,50 \%)\end{array}$ & $\begin{array}{l}P \\
\text { value }\end{array}$ \\
\hline Lung lesions, n (\%) & $12(30.0 \%)$ & $22(55.0 \%)$ & 0.024 \\
\hline Discharged, n (\%) & $40(100.0 \%)$ & 38 (95.0\%) & \multirow[t]{2}{*}{0.494} \\
\hline Died, n (\%) & $0(0.0 \%)$ & $2(5.0 \%)$ & \\
\hline $\begin{array}{l}\text { Hospitalization time (days, } x \\
\pm \text { sd) }\end{array}$ & $17.85 \pm 6.612$ & $20.72 \pm 5.01$ & 0.032 \\
\hline
\end{tabular}

All the matched 40 patients in the LH treatment group recovered and discharged from Huoshenshan hospital, in the usual treatment group, 38 patients discharged and 2 died (Table 3). Total mortality of matched 80 patients was $2.5 \%$ in this study. There was no significant difference for the proportion of discharged patients between two groups $(100.0 \%$ vs. $95.0 \%, P=0.494)$. Patients hospitalization time was significantly shorter in LH treatment group (17.85 \pm 6.612 days) compared with usual treatment group (20.72 \pm 5.01 days, $P=0.032)$. Further analysis of hospitalization time by Gehan-Breslow-Wilcoxon test indicated patients had shorter hospitalization time in LH treatment group $(P=0.0069)$ (Fig. 2).

\section{Secondary Outcomes}

After treatment, 12 (30\%) of 40 patients had lung lesions on chest CT in LH group, which is significantly lower than usual treatment group $(55.0 \%, \mathrm{P}=0.024)$ (Table 3$)$. The patients' laboratory tests after treatment were list in Table 4, smaller proportion of abnormal coagulation function was found in $\mathrm{LH}$ treatment group compared with usual treatment group $(27.5 \%$ vs. $50.0 \%, \mathrm{P}=0.039)$. The blood glucose level of patients in LH treatment group was higher than usual treatment group (5.50 \pm 1.172 vs. $4.67 \pm$ $0.774 \mathrm{mmol} / \mathrm{L}, \mathrm{P}=0.008)$. Other laboratory examination results were not found significantly differences. $22(55.0 \%)$ of 40 patients in LH group and 30 (75.0\%) in usual treatment group suffered from anemia, which might be associated with the patients' age. 
Table 4

Differences of laboratory indexes between two groups.

\begin{tabular}{|c|c|c|c|c|}
\hline & $\begin{array}{l}\text { Normal } \\
\text { range }\end{array}$ & $\begin{array}{l}\text { LH treatment } \\
\text { group }(n=40,50 \%)\end{array}$ & $\begin{array}{l}\text { Usual treatment } \\
\text { group }(n=40,50 \%)\end{array}$ & $\begin{array}{l}P \\
\text { value }\end{array}$ \\
\hline Leucocytes $\left(\times 10^{9} / L, x \pm s d\right)$ & $3.5-9.5$ & $5.52 \pm 1.586$ & $6.71 \pm 4.594$ & 0.202 \\
\hline$n(\%)$ & $\varangle 3.5$ & $2(5.0 \%)$ & $5(12.5 \%)$ & 0.432 \\
\hline$n(\%)$ & $\nabla 9.5$ & $1(2.5 \%)$ & $3(7.5 \%)$ & 0.615 \\
\hline Neutrophils ( $\left.\times 10^{9} / \mathrm{L},{ }^{\prime} x \pm s d\right)$ & $1.8-6.3$ & $3.38 \pm 1.269$ & $4.44 \pm 4.061$ & 0.194 \\
\hline $\begin{array}{l}\text { Lymphocytes ( } \times 10^{9} / \mathrm{L}, ' \mathrm{X} \pm \\
\text { sd) }\end{array}$ & $1.1-3.2$ & $1.50 \pm 0.461$ & $1.47 \pm 0.475$ & 0.814 \\
\hline$n(\%)$ & $\nabla 1.1$ & $8(20.0 \%)$ & $8(20.0 \%)$ & 1.000 \\
\hline Platelets $\left(\times 10^{9} / \mathrm{L}, \times \pm \mathrm{sd}\right)$ & $\begin{array}{l}125- \\
350\end{array}$ & $240.06 \pm 58.897$ & $225.14 \pm 95.282$ & 0.471 \\
\hline Haemoglobin (g/L, 'x $x \pm s d)$ & $\begin{array}{l}130- \\
175\end{array}$ & $123.88 \pm 11.542$ & $119.86 \pm 19.539$ & 0.340 \\
\hline$n(\%)$ & $\$ 130$ & $22(55.0 \%)$ & $30(75.0 \%)$ & 0.061 \\
\hline $\begin{array}{l}\text { Abnormal coagulation } \\
\text { function, } n(\%)\end{array}$ & NA & $11(27.5 \%)$ & $20(50.0 \%)$ & 0.039 \\
\hline Albumin (g/L, 'x $\pm s d)$ & $40-55$ & $37.19 \pm 3.282$ & $35.65 \pm 3.808$ & 0.124 \\
\hline $\begin{array}{l}\text { Alanine Aminotransferase } \\
\text { (IU/L, } x \pm s d)\end{array}$ & $9-50$ & $23.11 \pm 15.419$ & $71.27 \pm 191.903$ & 0.214 \\
\hline $\begin{array}{l}\text { Aspartate Aminotransferase } \\
\text { (IU/L, } x \pm \text { sd) }\end{array}$ & $15-40$ & $22.01 \pm 10.279$ & $52.18 \pm 149.878$ & 0.306 \\
\hline $\begin{array}{l}\text { Total bilirubin } \\
\text { (umol/L, 'x } \pm s d \text { ) }\end{array}$ & $0-26$ & $9.04 \pm 2.845$ & $11.50 \pm 16.036$ & 0.448 \\
\hline $\begin{array}{l}\text { Blood urea nitrogen } \\
\text { (mmol/L, 'x } x \mathrm{sd} \text { ) }\end{array}$ & $3.6-9.5$ & $5.26 \pm 1.941$ & $5.66 \pm 2.272$ & 0.523 \\
\hline $\begin{array}{l}\text { Serum creatinine } \\
\text { (umol/L, 'x } x \pm s d \text { ) }\end{array}$ & $57-111$ & $73.42 \pm 23.445$ & $73.97 \pm 22.574$ & 0.942 \\
\hline $\begin{array}{l}\text { Creatine kinase (IU/L, 'x } x \pm \\
\text { sd) }\end{array}$ & $24-190$ & $42.40 \pm 15.246$ & $40.69 \pm 18.394$ & 0.735 \\
\hline $\begin{array}{l}\text { Lactate dehydrogenase } \\
\text { (IU/L, 'X } \mathrm{X} \pm \mathrm{sd} \text { ) }\end{array}$ & $\begin{array}{l}120- \\
250\end{array}$ & $195.51 \pm 67.037$ & $216.54 \pm 186.569$ & 0.610 \\
\hline
\end{tabular}




\begin{tabular}{|c|c|c|c|c|}
\hline & $\begin{array}{l}\text { Normal } \\
\text { range }\end{array}$ & $\begin{array}{l}\text { LH treatment } \\
\text { group }(n=40,50 \%)\end{array}$ & $\begin{array}{l}\text { Usual treatment } \\
\text { group }(n=40,50 \%)\end{array}$ & $\begin{array}{l}P \\
\text { value }\end{array}$ \\
\hline $\begin{array}{l}\text { Myohemoglobin (ng/ml,'x } \mathrm{x} \\
\text { sd) }\end{array}$ & $0-80$ & $5.76 \pm 3.829$ & $8.86 \pm 2.937$ & 0.134 \\
\hline $\begin{array}{l}\text { Blood glucose } \\
\text { (mmol/L, } x \pm s d)\end{array}$ & $\begin{array}{l}3.9- \\
6.11\end{array}$ & $5.50 \pm 1.172$ & $4.67 \pm 0.774$ & 0.008 \\
\hline $\begin{array}{l}\text { C-reactive protein } \\
\text { (mg/L, 'x } x \mathrm{sd} \text { ) }\end{array}$ & $0-4$ & $4.54 \pm 5.835$ & $10.51 \pm 25.453$ & 0.244 \\
\hline n (\%) & $\varangle 4$ & $12(30.0 \%)$ & $10(25.0 \%)$ & 0.617 \\
\hline
\end{tabular}

\section{Discussion}

Like SARS-CoV and Middle East respiratory syndrome coronavirus (MERS-CoV), SARS-CoV-2 is a member of the $\beta$-coronavirus family. Pathological researches found SARS-CoV-2 could potently bind with angiotensin converting enzyme 2 (ACE2), a receptor abundantly expressed in a variety of human organ cells[22], which might account for multiple adverse effects in COVID-19 patients, such as lung, nervous system, kidney, liver, gastrointestinal system and heart[23-26]. There are no validated effective therapeutic approaches for COVID-19 patients, and the main treatment interventions are supportive therapy, including oxygen therapy, antiviral medications, antibiotics, corticosteroids and traditional Chinese medications[27]. The efficacy of antiviral medications that have been used for SARS and MERS is needed further explore in COVID-19 patients[28]. Moreover, more effective therapy agents and specific vaccines for COVID-19 are urgent needed to be validated by researchers. Zhu and colleagues have developed the recombinant adenovirus type-5 (Ad5) vectored COVID-19 vaccine[15]. The first-in-human trail were performed in 108 healthy adults, and results showed antibodies increased significantly and specific T-cell response was activated after vaccination[15], and the further phase of clinical trials are still ongoing.

Traditional Chinese medications have been proved to play a critical role in the prevention and treatment of several virus diseases, such as influenza virus, herpes simplex virus type 1 (HSV-1), SARS, and hepatitis B virus[29-31]. In clinical practices, traditional Chinese medications participated in patients' treatment collectively and were employed according to syndrome differentiation and stage of disease. In the pandemic of SARS-CoV-2, current treatment results have suggested the prescription of traditional Chinese medications are likely to be effective for COVID-19 pneumonia patients[32]. Li et al. reported a family cluster of 3 patients diagnosed with COVID-19 were treated with Chinese traditional patent medicine, Shuanghuanglian oral liquid, due to poor response to other therapeutic medications, and rapidly recovered without obvious adverse effects[33]. According to the report from National Health Commission of China, as for February $17,2020,85.20 \%$ of total 60,107 confirmed COVID-19 patients had received traditional Chinese medications. Based on the clinical efficiency and safety, traditional Chinese medications are recommended in the pneumonia diagnosis and treatment plan for new coronavirus 
infection (trial version 5) of the National Health Commission of the People's Republic of China. But reasonable large sample clinical trials are still necessary to confirm the treatment effects of traditional Chinese medications in this epidemic.

LH capsule is a classical patented traditional Chinese medication formula, which consists of 11 natural herbs including Lonicera japonica, Ephedra sinica, Isatis indigotica, Forsythia suspense, Pogostemon cablin, Rheum palmatum, Glycyrrhiza, Dryopteris crassirhizoma, Rhodiola crenulata, Houttuynia cordata, Prunus sibirica, gypsum and menthol as well as 61 chemical compounds[34]. The key components of Forsythia suspense has been proved to inhibit the expression of influenza M1 protein to block the spread of different influenza viruses in cell cultures[35]. Lonicera japonica could relieve sepsis-induced suppression of immune system by inhibiting lymphocyte apoptosis and promoting Th1 cytokine expression[36], and suppress toll-like receptor (TLR) signaling pathways in sepsis mouse model[37]. Rhodiola crenulata could ameliorate the hypoxia-induced endothelial cell death and oxidative stress by activating AMP-activated kinase (MAPK) and extracellular signal-regulated kinase 1/2 (ERK) signaling pathways[38]. Besides, Rheum palmatum is found to block the interaction of SARS-CoV spike protein and ACE2 in host cells[39] and against coxsackie virus (CV) and respiratory syncytial virus (RSV) in the early infection period[40]. In mouse model, Yang et al. found LH capsules inhibited inflammatory response and alleviated pathological impacts of lungs to reduce influenza B virus infection[41]. For influenza A (H1N1) patients, LH capsules showed similar antiviral clinical efficiency in the duration of illness and well toleration when compared with Oseltamivir in a randomized clinical trial[42]. In addition, recent study found that LH capsules exerted anti-coronavirus effect by reducing the virus load of SARS-CoV-2 and inhibiting the cytokine release in host cells[19]. COVID-19 patients treated with LH capsules have showed shorter duration of critical symptoms with reliable clinical safety[20]. These results have provided the evidence about the antiviral effects of LH capsules for clinical application.

In this study, we take a single center PSM analysis to evaluate the clinical efficacy and outcomes of LH capsules for the treatments of COVID-19 patients. Patients in LH capsules treatment group showed markedly shorter hospitalization time and higher discharge rate compared with that in usual treatment group. Besides, higher recovery rate of lung lesions and coagulation function in LH treatment group might also be relevant to antiviral activity. No statistical significance was found for the difference in the mortality and other laboratory indexes. The limitations of our study are as following: firstly, the sample size of patients in this study is small, and some critical statistical differences might not be apparent, such as mortality. Secondly, for the design of retrospective research, we did not classify the patients as mild and severe conditions when estimated the recovery time for the lack of relevant data. Thirdly, Wuhan Huoshenshan hospital is a makeshift hospital constructed during the outbreak of SARS-CoV-2. The hospitalized patients were transferred from other hospitals in Wuhan, and the variables of patients were significantly huge. Therefore, we established rigorous recruit criteria and performed PSM analysis to balance the baseline characteristics of $\mathrm{LH}$ treatment and comparator groups.

\section{Conclusion}


In conclusion, LH capsules have been widely used in clinical practice to against virus infection as antiviral agents. Here, we demonstrated the clinical effects of LH capsules by shortening the duration time of hospitalization and improving the recovery of chest CT manifestations in COVID-19 patients. Treatment with LH capsules might be considered for patients with COVID-19. Moreover, appropriately designed randomized trials in large cohort population are recommended to fully estimate the efficacy of LH capsules for SARS-CoV-2.

\section{Abbreviations}

\section{COVID-19}

Coronavirus disease 2019; LH:Lianhuaqingwen; SARS-CoV-2:Severe acute respiratory syndrome coronavirus 2; CT:computerized tomography; WHO:World Health Organization; ICU:Intensive care unit; PSM:Propensity score matching; MERS-CoV:Middle East respiratory syndrome coronavirus; ACE2:Angiotensin converting enzyme 2; HSV-1:Herpes simplex virus type 1; TLR:Toll-like receptor; $\mathrm{CV}$ :Coxsackie virus; RSV:Respiratory syncytial virus.

\section{Declarations}

\section{Acknowledgements}

Not applicable.

\section{Authors' contribution}

ZL, SZ: study design; ZL, YD, JK, XW, FZ: patient recruitmentandclinical data extraction; ZL, FZ, RL: dataanalysis; $Y D, F Z$, RL: data interpretation; $Z L, Y D, J K, X W, F Z$, RL: original draft preparation. All authors revised and approved the finalversion of the manuscript.

\section{Funding}

The research was supported by the technology and research projects fund of 920th Hospital (2019YGB20).

\section{Availability of data and materials}

The datasets supporting the results of this study are available from the corresponding author.

\section{Ethics approval and consent to participate}

This study was approved bythe ethic committee of Wuhan Huoshenshan Hospital and 920th Hospital of the PLA Joint Logistics Support Force. Informed consent was obtained from all patients for being included in the study. 
All authors critically reviewed the content of the manuscript. The consent for publication was obtained from all authors.

\section{Competing interests}

The authors declare no conflicts of interest.

\section{Author details}

${ }^{1}$ Department of Gastroenterology, 920th Hospital of the PLA Joint Logistics Support Force, Kunming,Yunnan650032, China. ${ }^{2}$ Department of Gastroenterology, Zhongshan hospital, Fudan university, Shanghai 200000, China. ${ }^{3}$ Wuhan Huoshenshan Hospital, Wuhan,Hubei 430000, China

\section{References}

1. Huang C, Wang Y, Li X, Ren L, Zhao J, Hu Y, Zhang L, Fan G, Xu J, Gu X, et al. Clinical features of patients infected with 2019 novel coronavirus in Wuhan, China. Lancet. 2020;395(10223):497-506.

2. Cao Y, Liu X, Xiong L, Cai K. Imaging and clinical features of patients with 2019 novel coronavirus SARS-CoV-2: A systematic review and meta-analysis. J Med Virol. 2020.

3. Wang D, Hu B, Hu C, Zhu F, Liu X, Zhang J, Wang B, Xiang H, Cheng Z, Xiong Y, et al. Clinical Characteristics of 138 Hospitalized Patients With 2019 Novel Coronavirus-Infected Pneumonia in Wuhan, China. Jama. 2020;323(11):1061-9.

4. Lu R, Zhao X, Li J, Niu P, Yang B, Wu H, Wang W, Song H, Huang B, Zhu N, et al. Genomic characterisation and epidemiology of 2019 novel coronavirus: implications for virus origins and receptor binding. Lancet. 2020;395(10224):565-74.

5. Zhang G, Zhang J, Wang B, Zhu X, Wang Q, Qiu S. Analysis of clinical characteristics and laboratory findings of 95 cases of 2019 novel coronavirus pneumonia in Wuhan, China: a retrospective analysis. Respir Res. 2020;21(1):74.

6. Yang L, Liu J, Zhang R, Li M, Li Z, Zhou X, Hu C, Tian F, Zhou F, Lei Y. Epidemiological and clinical features of 200 hospitalized patients with corona virus disease 2019 outside Wuhan, China: A descriptive study. J Clin Virol. 2020;129:104475.

7. Yang X, Yu Y, Xu J, Shu H, Xia J, Liu H, Wu Y, Zhang L, Yu Z, Fang M, et al. Clinical course and outcomes of critically ill patients with SARS-CoV-2 pneumonia in Wuhan, China: a single-centered, retrospective, observational study. Lancet Respir Med. 2020;8(5):475-81.

8. Lu CC, Chen MY, Lee WS, Chang YL. Potential therapeutic agents against COVID-19: What we know so far. J Chin Med Assoc. 2020;83(6):534-6.

9. Gordon CJ, Tchesnokov EP, Feng JY, Porter DP, Götte M. The antiviral compound remdesivir potently inhibits RNA-dependent RNA polymerase from Middle East respiratory syndrome coronavirus. J Biol Chem. 2020;295(15):4773-9. 
10. Mulangu S, Dodd LE, Davey RT Jr, Mbaya T, Proschan O, Mukadi M, Lusakibanza Manzo D, Nzolo M, Tshomba Oloma D, Ibanda A. A et al. A Randomized, Controlled Trial of Ebola Virus Disease Therapeutics. N Engl J Med. 2019;381(24):2293-303.

11. Holshue ML, DeBolt C, Lindquist S, Lofy KH, Wiesman J, Bruce H, Spitters C, Ericson K, Wilkerson S, Tural A, et al. First Case of 2019 Novel Coronavirus in the United States. N Engl J Med. 2020;382(10):929-36.

12. Vincent MJ, Bergeron E, Benjannet S, Erickson BR, Rollin PE, Ksiazek TG, Seidah NG, Nichol ST. Chloroquine is a potent inhibitor of SARS coronavirus infection and spread. Virol J. 2005;2:69.

13. Gao J, Tian Z, Yang X. Breakthrough. Chloroquine phosphate has shown apparent efficacy in treatment of COVID-19 associated pneumonia in clinical studies. Biosci Trends. 2020;14(1):72-3.

14. Zhai P, Ding Y, Wu X, Long J, Zhong Y, Li Y. The epidemiology, diagnosis and treatment of COVID-19. Int J Antimicrob Agents. 2020;55(5):105955.

15. Zhu FC, Li YH, Guan XH, Hou LH, Wang WJ, Li JX, Wu SP, Wang BS, Wang Z, Wang L, et al. Safety, tolerability, and immunogenicity of a recombinant adenovirus type- 5 vectored COVID-19 vaccine: a dose-escalation, open-label, non-randomised, first-in-human trial. Lancet. 2020;395(10240):1845-54.

16. Lee N, McGeer A. The starting line for COVID-19 vaccine development. Lancet. 2020;395(10240):1815-6.

17. Ding Y, Zeng L, Li R, Chen Q, Zhou B, Chen Q, Cheng PL, Yutao W, Zheng J, Yang Z, et al. The Chinese prescription lianhuaqingwen capsule exerts anti-influenza activity through the inhibition of viral propagation and impacts immune function. BMC Complement Altern Med. 2017;17(1):130.

18. Zhang ZJ, Morris-Natschke SL, Cheng YY, Lee KH, Li RT. Development of anti-influenza agents from natural products. Med Res Rev. 2020.

19. Runfeng L, Yunlong H, Jicheng H, Weiqi P, Qinhai M, Yongxia S, Chufang L, Jin Z, Zhenhua J, Haiming $\mathrm{J}$, et al. Lianhuaqingwen exerts anti-viral and anti-inflammatory activity against novel coronavirus (SARS-CoV-2). Pharmacol Res. 2020;156:104761.

20. Hu K, Guan WJ, Bi Y, Zhang W, Li L, Zhang B, Liu Q, Song Y, Li X, Duan Z, et al. Efficacy and safety of Lianhuaqingwen capsules, a repurposed Chinese herb, in patients with coronavirus disease 2019: A multicenter, prospective, randomized controlled trial. Phytomedicine. 2020:153242.

21. Morgan CJ. Reducing bias using propensity score matching. J Nucl Cardiol. 2018;25(2):404-6.

22. Bourgonje AR, Abdulle AE, Timens W, Hillebrands JL, Navis GJ, Gordijn SJ, Bolling MC, Dijkstra G, Voors AA, Osterhaus AD, et al. Angiotensin-converting enzyme 2 (ACE2), SARS-CoV-2 and the pathophysiology of coronavirus disease 2019 (COVID-19). J Pathol. 2020.

23. Jiang F, Deng L, Zhang L, Cai Y, Cheung CW, Xia Z. Review of the Clinical Characteristics of Coronavirus Disease 2019 (COVID-19). J Gen Intern Med. 2020;35(5):1545-9.

24. Jin X, Lian JS, Hu JH, Gao J, Zheng L, Zhang YM, Hao SR, Jia HY, Cai H, Zhang XL, et al. Epidemiological, clinical and virological characteristics of 74 cases of coronavirus-infected disease 2019 (COVID-19) with gastrointestinal symptoms. Gut. 2020;69(6):1002-9. 
25. Mo P, Xing Y, Xiao Y, Deng L, Zhao Q, Wang H, Xiong Y, Cheng Z, Gao S, Liang K, et al. Clinical characteristics of refractory COVID-19 pneumonia in Wuhan, China. Clin Infect Dis. 2020.

26. Wu Y, Xu X, Chen Z, Duan J, Hashimoto K, Yang L, Liu C, Yang C. Nervous system involvement after infection with COVID-19 and other coronaviruses. Brain Behav Immun. 2020;87:18-22.

27. Huang Q, Deng X, Li Y, Sun X, Chen Q, Xie M, Liu S, Qu H, Liu S, Wang L, et al. Clinical characteristics and drug therapies in patients with the common-type coronavirus disease 2019 in Hunan, China. Int $\mathrm{J}$ Clin Pharm. 2020;42(3):837-45.

28. Şimşek Yavuz S, Ünal S. Antiviral treatment of COVID-19. Turk J Med Sci. 2020;50(Si-1):611-9.

29. Li W, Wang XH, Luo Z, Liu LF, Yan C, Yan CY, Chen GD, Gao H, Duan WJ, Kurihara H, et al. Traditional Chinese Medicine as a Potential Source for HSV-1 Therapy by Acting on Virus or the Susceptibility of Host. Int J Mol Sci. 2018; 19(10).

30. Qi FH, Wang ZX, Cai PP, Zhao L, Gao JJ, Kokudo N, Li AY, Han JQ, Tang W. Traditional Chinese medicine and related active compounds: a review of their role on hepatitis $B$ virus infection. Drug Discov Ther. 2013;7(6):212-24.

31. Ai H, Wu X, Qi M, Zhang L, Hu H, Zhao Q, Zhao J, Liu H. Study on the Mechanisms of Active Compounds in Traditional Chinese Medicine for the Treatment of Influenza Virus by Virtual Screening. Interdiscip Sci. 2018;10(2):320-8.

32. Zhang L, Yu J, Zhou Y, Shen M, Sun L. Becoming a Faithful Defender: Traditional Chinese Medicine against Coronavirus Disease 2019 (COVID-19). Am J Chin Med. 2020;48(4):763-77.

33. Ni L, Zhou L, Zhou M, Zhao J, Wang DW. Combination of western medicine and Chinese traditional patent medicine in treating a family case of COVID-19. Front Med. 2020;14(2):210-4.

34. Wang CH, Zhong Y, Zhang Y, Liu JP, Wang YF, Jia WN, Wang GC, Li Z, Zhu Y, Gao XM. A network analysis of the Chinese medicine Lianhua-Qingwen formula to identify its main effective components. Mol Biosyst. 2016;12(2):606-13.

35. Law AH, Yang CL, Lau AS, Chan GC. Antiviral effect of forsythoside A from Forsythia suspensa (Thunb.) Vahl fruit against influenza A virus through reduction of viral M1 protein. $J$ Ethnopharmacol. 2017;209:236-47.

36. Kim SJ, Kim JS, Choi HS, Kim YM, Hong SW, Yeon SH, Kim Y, Lee SM. HS-23, a Lonicera japonica extract, reverses sepsis-induced immunosuppression by inhibiting lymphocyte apoptosis. J Ethnopharmacol. 2015;171:231-9.

37. Kim SJ, Yoon SJ, Kim YM, Hong SW, Yeon SH, Choe KI, Lee SM. HS-23, Lonicera japonica extract, attenuates septic injury by suppressing toll-like receptor 4 signaling. J Ethnopharmacol. 2014;155(1):256-66.

38. Chang PK, Yen IC, Tsai WC, Chang TC, Lee SY. Protective Effects of Rhodiola Crenulata Extract on Hypoxia-Induced Endothelial Damage via Regulation of AMPK and ERK Pathways. Int J Mol Sci. $2018 ; 19(8)$.

39. Ho TY, Wu SL, Chen JC, Li CC, Hsiang CY. Emodin blocks the SARS coronavirus spike protein and angiotensin-converting enzyme 2 interaction. Antiviral Res. 2007;74(2):92-101. 
40. Liu Z, Ma N, Zhong Y, Yang ZQ. Antiviral effect of emodin from Rheum palmatum against coxsakievirus B5 and human respiratory syncytial virus in vitro. J Huazhong Univ Sci Technolog Med Sci. 2015;35(6):916-22.

41. Yang C, Wang Y, He J, Yan W, Jiang H, Chen Q, Li L, Yang Z. Lianhua-Qingwen Displays Antiviral and Anti-Inflammatory Activity and Synergistic Effects with Oseltamivir against Influenza B Virus Infection in the Mouse Model. Evid Based Complement Alternat Med. 2020; 2020:3196375.

42. Duan ZP, Jia ZH, Zhang J, Liu S, Chen Y, Liang LC, Zhang CQ, Zhang Z, Sun Y, Zhang SQ, et al. Natural herbal medicine Lianhuaqingwen capsule anti-influenza A (H1N1) trial: a randomized, double blind, positive controlled clinical trial. Chin Med J (Engl). 2011;124(18):2925-33.

\section{Figures}

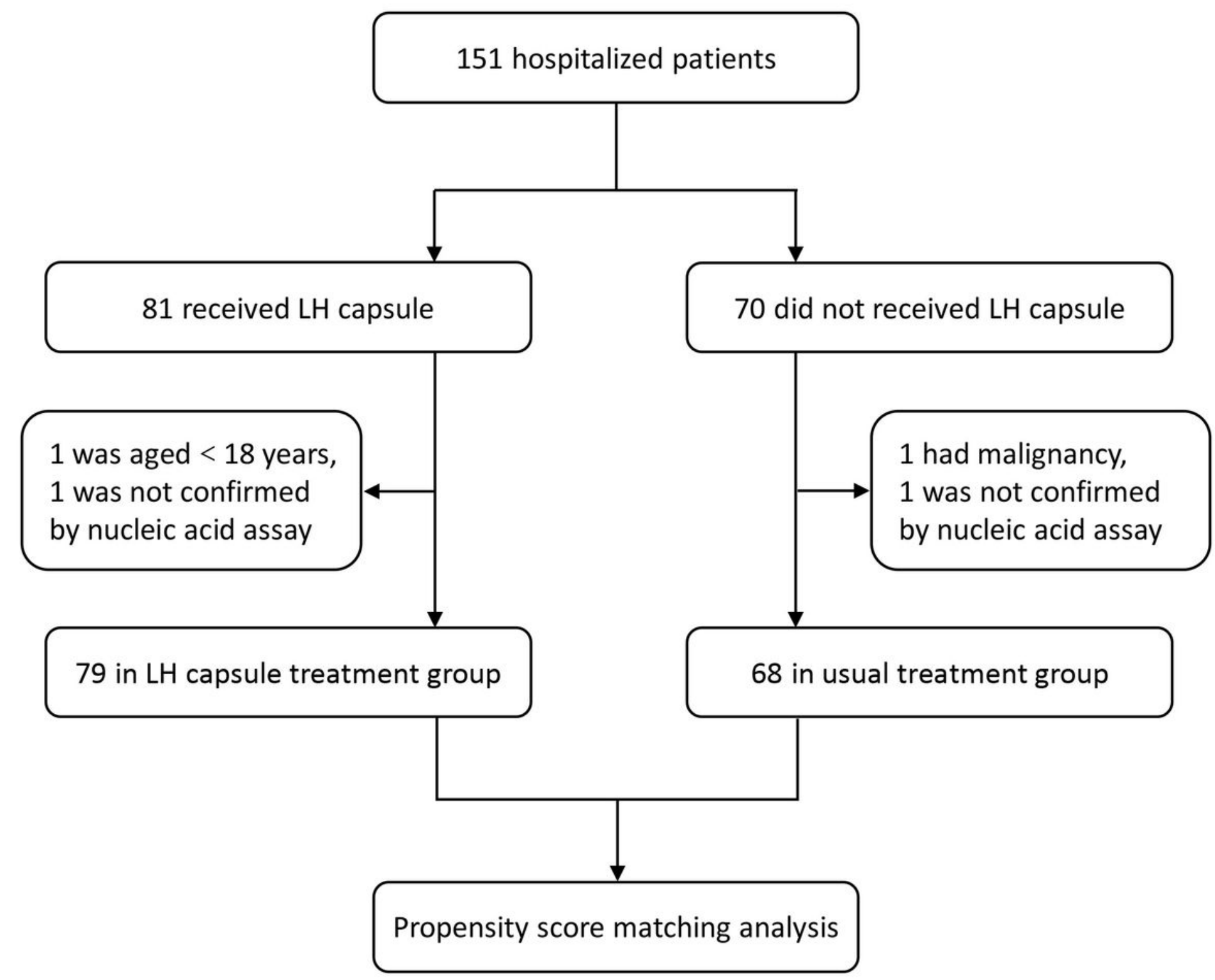

Figure 1 


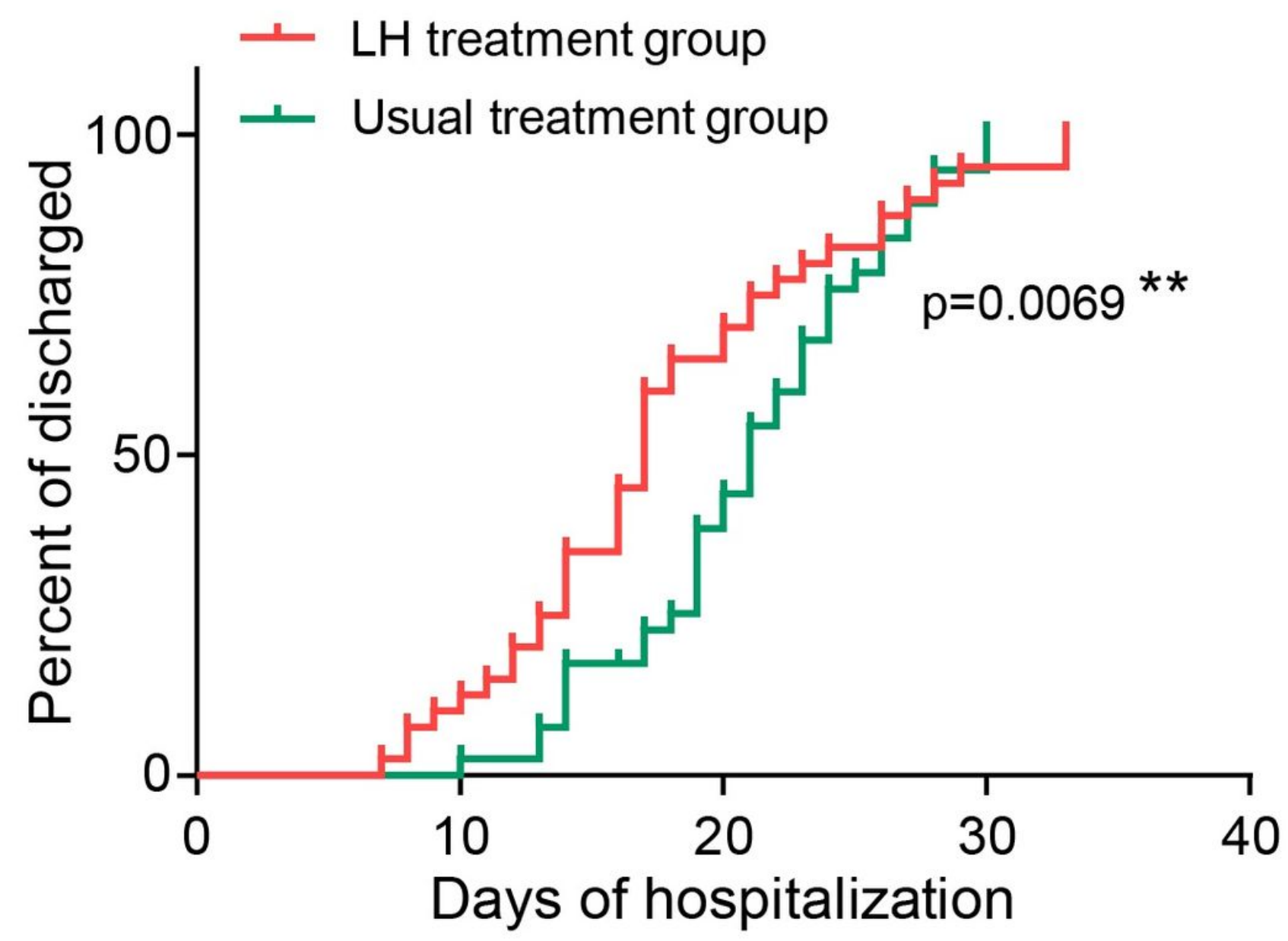

Number of discharged

\begin{tabular}{lcccc}
\hline Days & 0 & 10 & 20 & 30 \\
\hline LH treatment group & 40 & 5 & 28 & 38 \\
Usual treatment group & 40 & 1 & 19 & 40 \\
\hline
\end{tabular}

Figure 2

Comparation of the discharge rate between two groups. Two patients died in usual treatment group. 